\title{
Comprehensive treatment progress of metastatic bone tumor
}

\author{
Rui Jiang ${ }^{1}$, Ziyan Zhang ${ }^{2}$, Han $\mathrm{Wu}^{1}$ and Chao Zhang ${ }^{3,}$ a, * \\ ${ }^{1}$ Department of orthopedics, China-Japan Union Hospital of Jilin University, Jilin 130033, China \\ ${ }^{2}$ Department of orthopedics, The Second Hospital of Jilin University, Jilin 130041, China \\ ${ }^{3}$ Department of ophthalmology, The Second Hospital of Jilin University, Jilin 130041, China \\ azhangchao0701@163.com
}

Keywords: bone tumor, tumor metastasis, comprehensive therapy.

\begin{abstract}
Objective: to summarize and analyze the clinical characteristic and clinical features of metastatic bone tumors: metastatic bone tumors occur in male patients mostly, mainly including male patients from 41 to 70 years old. The most common primary lesions of male patients include lung, prostate and liver. The most common primary lesions of male patients include lung, breast, stomach and intestine and unknown origin. Early single lesion is mostly discovered in the spine and pelvis. Late lesion is mostly metastatic in many parts of the body. Patients are often diagnosed due to physical pain (swelling and dysfunction), pathological fracture and even paraplegia, etc.. Some patients are diagnosed due to symptoms of primary lesions, and bone metastasis is discovered during diagnosis examination. Osteolytic change is commonly discovered in imageology. Treatment is based on symptomatic treatment, pain relief, chemotherapy and radiotherapy. Surgery is mostly palliative. Conclusion: the clinical features of metastatic bone tumors are complicated, and the pathologic features of metastatic bone tumors should be grasped for early diagnosis and treatment.
\end{abstract}

\section{Introduction}

Bone metastatic tumor ranks No. 1 among bone neoplasms. Tumors in breast, lung, prostate, kidney and thyroid are the most common. The morbidity of various metastatic tumors are related to the molecular biology characteristics on the one hand, it is also related to tissue characteristics of metastasis on the other hand. Other important influence factors include vascular access, blood flow, etc.. Although metastatic tumors of humerus and femur are more common, metastatic bone tumors are mainly distributed on axial skeleton, such as spine, pelvis and rib.

Metastatic bone tumor has the therapeutic goal of alleviating symptoms. Only a few tumors can be cured. External beam radiotherapy, chemotherapy, endocrinotherapy, radioisotope, etc. are important therapeutic methods. the destruction of skeletal structure, Orthopedist should treat the destruction of skeletal structure accordingly, and special treatment is needed for hypercalcemia.

\section{Supportive therapy of bone metastatic cancer}

Skeletal metastasis disease often results in many physiological and psychological problems. Pain, fatigue and mental stress are the most common symptoms. Pain is the most prominent manifestation. About $70 \%$ of metastatic bone tumor patients suffer pain during their course of illness.

\subsection{Ostealgia therapy}

Though pathogenesis of metastatic bone tumor has been well explained from studies in recent years, the etiology of skeleton pain is not clear at present. Effective therapy can not be achieved due to incomplete pain evaluation. Pain therapy should be established on the basis of comprehensive evaluation on pain, disease relationship and influence of pain and disease state on patient life quality. Pain description includes pain degree, nature and relieving factors. Pain and disease relationship can be made clear according to information obtained from disease history, physical examination, laboratory examination, imaging examination. Evaluation results are used for determining whether further evaluation is required or not, and they will affect the selection of therapeutic methods. Pain 
drug therapy should be individualized on the basis of careful consideration of patient pain type, social, psychological and economic factors. Treatment should begin with simple dose and minimal invasive method. When opioids are used for secondary pain killing, paracetamol should be merged and used mostly. When patients suffer from moderate to severe pain, although opium analgesic can be used, anesthetic and NSAID mentioned in third-level analgesic plan should be given at the same time. Therefore, anaesthetic can exert the maximum effect, which can not exceed maximum recommended dose of paracetamol, aspirin and other NSAID at the same time, thereby avoiding influence on implementation of secondary analgesic plan [1].

Oral medication is widely applied in long-acting opioids therapy. Some other administration routes are also frequently used due to vomiting, low gastroenteric function, and non-oral medication. In some intractable pain therapies, opioids can be administered through skin, rectum and continuous subcutaneous or intravenous injection and intramedullary injection.

\subsubsection{Primary paregoric}

NSAID can inhibit epoxidase system and block the production of prostaglandin and interleukin. These are important inflammatory factors, which can increase the the sensibility of nervous system to pain stimulation. Anti - inflammatory action of these drugs can mitigate pain caused by bone metastasis. However, if the drugs are overused, severe liver and kidney toxicity can be caused. Because NSAID does not act on the opioid receptor, it can be used together with the opioids, and its effect is better than joint application of two NSAID drugs. There are many prescription and non-prescription NSAID at present, the price and dosage vary greatly. Since paracetamol has weaker anti-inflammatory action than NSADI, curative effect to bone metastasis patients is relatively poor. Daily dosage should not exceed $4 \sim 6 \mathrm{~g}$ in order to prevent liver toxicity.

\subsubsection{Secondary pain killer}

Secondary pain killers include codeine, paracodin, hydrogenation codeine, oxycodone, propoxyphene, etc., which belong to quick and short-acting opioids. It is frequently used when moderate pain can not be controlled by general NSAID. Opioids, such as hydrogenation codeine and oxycodone, are frequently used together with aspirin, para-dimethylaminol, etc. Daily maximum dose should be controlled so that potential toxic reaction can not be caused. Dolantin is not suitable for treatment of chronic cancer pain. It can lead to central nervous system stimulation and shock due to shorter half-time and metabolite toxicity.

\subsubsection{Third-level pain killer}

When patients have moderate to severe pain, and secondary paregoric can not be used for controlling the pain, third-level pain killer should be used for treatment. It includes morphine, dihydromorphone, para-dimethylaminol and phenolphthalein. These drugs belong to pure opioid receptor antagonists, which are in linear dose-response relationship. Therapeutic dose should follow the individualized principle. Quick and short-acting oral opioids can be effective within half an hour after administration. The drug effect lasts for about four hours. If the pain occurs again soon, the dosage of the drug needs to be increased until the pain relief time reaches 4 hours. The starting dose of the drug varies with pain degree, whether opioids are used or not in the past, and different medical conditions. The starting dose is usually given subcutaneously or statically according to $5 \sim 10 \mathrm{mg} / 4 \mathrm{~h}$. The therapeutic dose is usually $5 \%$ to $15 \%$ of the daily dose. The minimum time interval for oral medication is $1.5 \sim 2$ hours. The primary drug function can be exerted as far as possible before next medication as a result. The minimum time interval is $10 \sim 15$ minutes for subcutaneous or intravenous injection.

\subsection{Tricyclic antidepressant}

both share now with opioids after $1 \sim 2$ weeks, The antidepressant onset time is earlier within one to two weeks after medication currently regardless of joint administration with opioids or not.

\subsection{Anticonvulsants}

anticonvulsants has better effect in treating stabbing pain and neuralgia. It can be used separately or jointly with tricyclic antidepressants. Gabapentin is an excellent drug for treating pain caused by intractable neuropathic cancers. Valproic acid and clonazepam can strengthen the inhibitory effect of neurons, thus effectively treating neuralgia. 


\subsection{Corticosteroids}

since corticosteroids can be used for improving patient mood, increasing appetite, and reducing inflammation and nausea, it can be used in metastatic bone tumor therapy. It can reduce pain caused by peripheral nerve edema and nerve compression. It is a routine drug for patients with pain caused by spinal cord compression. Dexamethasone can pass through the blood-brain barrier. The half-time is longer. Therefore, hormone drugs are the best choice for patients with pain caused by spinal cord compression.

\section{Hypercalcemia therapy}

Hypercalcemia affects $10 \%$ to $40 \%$ cancer patients, especially for squamous cell carcinoma (head and neck, esophagus, lung and bladder), breast, kidney and ovarian cancers as well as multiple cutaneous leiomyoma. The common complication symptoms of hypercalcemia include anorexia, nausea, vomiting, hyperuria, dehydration, constipation, etc. Delirium is also a common complication, which can be developed into slow motion and coma. There are generally three possible mechanisms for malignant tumor patients to produce hypercalcemia. Humoral hypercalcemia of malignancy of malignant tumor is mainly caused by tumor by-products, such as biological medium parathyroid hormone related protein ( PT HrP) secreted in breast cancer, renal cell carcinoma and squamous cell carcinoma, which can activate the bone metabolism, thereby resulting in activity increase of osteoclast.

Calcium absorption can be increased due to decrease of kidney's removal of calcium aiming at most hypercalcemia patients. PTHrP can act on the hormone receptors of the parathyroid glands to increase the reabsorption of renal tubules to calcium. In addition, hypercalcemia can reduce the concentration ability of renal tubules, thereby resulting in diuresis and decrease of blood volume. Hypercalcemia patient should undergo intravenous fluids, thereby correcting blood volume decline, and increasing the filtration rate of glomerular and calcium secretion of the kidney.

Massive intravenous fluid infusion and application of urinalysis can increase the urine, the functions thereof are limited, and risks can be caused. The drug should be rarely used, or it cannot be used. Corticosteroids are effective only for tumors sensitive to corticosteroids. It should be used at low dosage or used separately. Ticyclic antidepressant is particularly effective for treatment of neuropathic pain, which can enhance the effect of opioids, and it has certain analgesic effect itself. Because it can stimulate emotion and has sedative effect, the drug can be used for assisting patients with metastasis tumour and depression. Amitriptyline is a tricyclic antidepressant with the most application. The patients can start with $25 \mathrm{mg}$ before sleep, and the dose can be gradually increased until excellent painkilling effect is obtained. Dispramine and nortriptyline also can be used for treating nerve pain well according to proof, and the toxic side effects can be reduced [2].

Calcitonin is an osteoclast inhibitor that can rapidly reduce the calcium ion concentration in plasma, but its effect is short. Therefore, it only can be used during urgent reduction of calcium ion concentration, and it is used for the initial therapy in serious hypocalcemia. Bisphosphonate treatment is the main treatment means for resisting hypercalcemia. Intravenous administration of pamidronate is very effective, and it is well tolerated, and the plasma calcium ion concentration can be restored to normal level aiming at $70 \% \sim 100 \%$ patients with hypercalcemia due to tumor. Calcium ion concentration begins to decline $24 \sim 48$ hours after application of pamidronate, which can be returned to normal level $3 \sim 5$ days later. Normal calcium ion level can last for several days to several months. In a dose-comparison study, the dose-response relationship thereof has been known. Namely pamidronate dose is larger, the function is stronger. The recommended pamidronate dose is $60 \sim 90$ $\mathrm{mg}$, which is intravenously administered slowly in 4 hours. Plasma calcium ion concentration, phosphate and electrolyte should be monitored at any time. However, the best therapeutic method for tumor-induced hypercalcemia is effective treatment of primary malignant tumor. Hypercalcemia control is the only choice due to insufficient effective anticancer drug method. The patient should be 
advised to maintain adequate fluid intake. Patients should move as much as possible and receive treatment suitably during hypercalcemia relapse.

\section{Non-operative treatment of bone metastatic cancer}

\subsection{Systemic treatment}

The most effective and practical systemicanti-tumor therapy should be given firstly in order to treat patients with bone metastasis tumors. Systemic treatment with different alternative therapies can block the effects of tumor material on host cells. Chemotherapy, endocrinotherapy and bone isotope therapies can play the anti-tumor role. Bisphosphonate can prevent host cells (osteoblasts) from reacting to cancerogenic substance. Usually, the systemic treatment of malignant tumor skeleton metastasis is the same as that of metastasis cancer therapy in other parts, which depends on the tumor type. Metastasis of breast cancer and prostate cancer plays an important role. Firstly, the systemictherapy to the above tumors is comparatively effectively. Secondly, since metastasis of these two tumors accounts for most proportion among bone metastatic tumors, appropriate paregoric or auxiliary therapeutic methods are required for ostealgia caused by bone metastasis aiming at tumors which can not be treated effectively and easily.

\subsection{Radioisotope therapy}

The clinical application of bone radioisotope therapy is based on medium to high energy isotope. It reaches skeletons with tumor involvement in a targeted mode selectively, which can effectively play the anti-tumor role, and radiation injury to normal tissue can be avoided. The use of radioisotope is also inhibited since it has the toxicity function restricting bone marrow growth.

\subsection{External irradiation}

Radiotherapy is the first choice for relieving pain in individual parts. The body study shows that radiotherapy can change the structure of metastasis focus skeleton. Firstly, degeneration and necrosis of tumor cells are caused, and then the proliferation of collagen can be produced, thereby resulting in fibrous interstitium of rich blood vessels. Osteoblasts can form new braided bones under the circumstance. The interstitial mass in the bone trabecula will be replaced by the bone marrow tissue. Calcification occurs again in the areas with bone destruction within 3 to 6 weeks after irradiation. Calcification reaches its peak in $2 \sim 3$ months. Once the patient decides to take radiotherapy, other factors must be considered, such as how to adjust the fixed frame (if necessary), radiation dose, therapy duration, whether it is necessary to use with other therapy means or not, systemic radioisotope therapy, etc. The choice of therapeutic dose and duration depends on many factors, including the expectation of life, functional status, the number of metastasis focuses, the location and size of the therapy tissues [3-4].

Radiotherapy technology and dose vary widely in different medical centers, and there is no special method to show obvious advantages in pain relief. Random prospective study on more than 1000 cases of pain caused by therapy bone metastatic cancer has been reported, different doses are adopted, the dose can be $15 \mathrm{~Gy}$, which is irradiated for five times, or the dosage can be $40.5 \mathrm{~Gy}$, which can be irradiated for 15 times. The effective rate is about $85 \%$. Pain of $50 \%$ patients is completely relieved, and pain of $35 \%$ patients is partially relieved. The duration of complete pain relief is 12 to 15 weeks. 40. 5 Gy is applied, which is radiated for 15 times, pain of $35 \%$ patients is completely alleviated. 25 Gy is additionally adopted, which is irritated for 5 times, and pain of $28 \%$ patients can be completely alleviated. However, other randomized prospective studies indicate that there is no significant difference in the effect of single irradiation or subdivided continuous irradiation for two to three weeks.

Pain relief usually occurs very quickly, more than half of the patients feel effective within a week or two weeks. If the pain relief is not obvious 6 weeks or longer time after therapy, the pain can not be relieved again. It is necessary to use internal fixation to prevent fracture during mass focus radiotherapy. 
Two most important conditions for choosing appropriate orthopaedic surgical therapy are tumor type and site in addition to general medical conditions. The pathologic fracture following prostate cancer can be healed well even after large-dose radiation possibly because there are a large number of reactive bones in the focus area. Similarly, therapy of traditional method on pathologic fracture of breast cancer also has good healing potential. However, bone metastasis focus following lung cancer often causes destruction and dissolution of bone, and it is difficult to see osteogenic reaction. The pathological fracture of bone metastasis focus from thyroid cancer usually has better potential healing ability generally after proper restriction of activity. If there is no surgical indication, radiotherapy, endocrinotherapy and chemotherapy can be jointly used aiming at the skeleton focus from thyroid cancer usually, thereby achieving spontaneous ossification in the focus site. When surgical methods methods are not used as therapy, various orthopaedic splint techniques can be used according to metastasis sites. When patients with massive cervical vertebral destruction, accompanied by vertebral instability, are not suitable for surgery, support can be used for firm fixation, and auxiliary radiation is applied, and excellent heating effect can be obtained usually. The zonesthesia of focus in thoracic or lumbar sites can be alleviated [5].

Metastasis focus can reach acetabulum and proximal femur, which can be replaced by hip prosthesis. When focus metastasis reaches pelvis, the patient is not suitable for surgical therapy, crutches, canes, wheelchairs, etc. can be used for alleviating the burden of the pelvis. When metastasis focus is located in femur and tibia, surgery internal fixation can be used, methyl methacrylate can be used or not as reinforcement, patients can retain the ability to act independently. When the patient is unable to accept surgical therapy, splint and stent or therapy experience should be applied to ensure the tolerability of normal tissues, especially spinal cord. When external irradiation is more dangerous, radioisotope treatment is a good choice for relieving symptoms.

\subsection{External fixation and support}

Non-surgical orthopedic surgery therapy is necessary for bone metastatic cancer accompanied with bone destruction, hyperplasia and pathological fracture complication therapy. The therapy has the purpose of focusing on relieving pain, reducing the use of anaesthetic, preventing fracture of the weight-bearing skeleton, and allowing patients to restore normal functions and social activities completely as far as possible. It is unrealistic to implement radical surgery therapy aiming at the patients, and plastic splint can relieve pain to a certain extent.

\subsection{Bisphosphonates}

In recent years, bisphosphonate has gradually used to inhibit osteoclast. It is a mixture with P-C-P as the base unit. The chemical structure can prevent phosphorylation process, and its activity is greatly different according to the difference in fatty acid carbon chain skeleton length and structure. Bisphosphonate is combined to the surface of hydroxyapatite, biochemical effects are produced on the surface of its crystal, thereby causing physiological changes. The bone metabolism can be adjusted by inhibiting the activity of osteoclast. There have been many reports on the role of bisphosphonate in preventing bone damage recently. It is found that bisphosphonate can effectively reduce skeleton metastasis and ostealgia of multiple cutaneous leiomyoma and breast cancer. Pamidronate is given through vein pathway once every $3 \sim 4$ weeks. The skeleton complication of bone metastasis breast cancer patients can be prominently reduced continuously 24 months later. Pamidronate shows its good safety and tolerability aiming at both breast cancer or cutaneous leiomyoma patients. Whether similar therapy also can be used for other skeleton metastasis caused by malignant tumor or not, lung cancer and kidney cancer patients accompanied with osteoclastic bone metastasis focus may benefit from the therapy. Prostate cancer patients with progressive bone metastasis patient frequently suffer from severe ostealgia in the process of endocrinotherapy. Since effective chemical therapy is not available, it is still a huge challenge at present. Although the skeleton metastasis often has osteogenesis behavior simultaneously, histopathologic slide suggests that the activity of osteoclast also can be increased under the condition, while the bisphosphonate can relieve pain. Bisphosphonate therapy plays a role for breast cancer women with no skeleton metastasis. Anticancer drug of breast cancer patients often lead to gonadopause, which not only can increase the risk of skeleton metastasis, 
but also can cause early amenorrhea osteoporosis. Bisphosphonate treatment is adopted for benefiting two aspects of patients.

\section{Surgical therapy}

The primary tumor types should be determined, the patient condition and the functional expectation should be evaluated, surgery with lower tendency invasiveness (such as vertebral plasty) or non-surgical therapy should be adopted for patients with shorter expected survival time or patients with more severe medical diseases, the risks and complications are discussed and evaluated with patients and family members together, and the possible functions after reconstructive surgery are informed to patients [6-7].

\section{Acknowledgments}

This work was supported by the study of c-Myc and m TOR inhibitors in the combined targeting therapy of osteosarcoma. Jilin provincial finance department (No 3D517P373430).

\section{References}

[1]. Weis LD.Adolesc Med.1999,10 (3):451 453.

[2]. Feng Chuanhan. Progress of bone tumor diagnosis therapy. Chinese Journal of Orthopaedics, 2000,20 (2) supplementary issue: 10.

[3]. Aberg M,Rydholm A,Holmberg J,et al.Reconstruction with a free Vascularized fibular.graft for malignant bone tumor.Acta Orthop scand [J].1988,59:430 437.

[4]. Finkemeier CG Bone-grafting and bone-graft substitutes J Bone Joint Surg (Am), 2002, 84 (3):454 464.

[5]. Guo Weizhu [translator]. Bone and soft tissue tumor diagnosis and therapy. Tianjin: Tianjin Technology Translation Publishing Co., Ltd. ,2007,1: 154.

[6]. Delaney TF,Trofimov AV,Engelsman M,et al.Adancedtechnology radiation therapy in the management of bone and soft tissue sarcomas.Cancer Control,2005,12:27 35.

[7]. Cai Zhengdong, Ji Fang, Practical bone oncology. Beijing: People's Military Medical Publishing House. 2004,8:154- 155. 